\title{
Sensitivity of reaction cross sections to halo nucleus density distributions
}

\author{
M. P. Bush, J. S. Al-Khalili, J. A. Tostevin, and R. C. Johnson \\ Department of Physics, University of Surrey, Guildford, Surrey, GU2 5XH, United Kingdom
}

(Received 9 November 1995)

\begin{abstract}
Measurements of reaction cross sections are routinely used to deduce effective nuclear root mean square (rms) radii by comparison with theoretical model predictions. Cross sections calculated using the optical limit Glauber model depend strongly on the rms radius of the density assumed for the projectile nucleus. We investigate such calculations by assuming a range of projectile density distributions. We show that calculated ${ }^{11} \mathrm{Li}$-target cross sections at fixed rms radii retain a significant sensitivity to higher radial moments of the projectile density which is quite different for light and heavy targets. [S0556-2813(96)02905-6]
\end{abstract}

PACS number(s): $24.10 . \mathrm{Ht}, 24.50 .+\mathrm{g}, 25.10 .+\mathrm{s}, 21.10 . \mathrm{Gv}$

\section{INTRODUCTION}

Since the first measurements of unusually large reaction cross sections $\left(\sigma_{R}\right)$ of neutron-rich light nuclei, there has been considerable discussion of the use of optical limit Glauber models $[1,2]$ to extract information about the density of such nuclei. Tanihata and coworkers $[3,4]$ found that large rms radii were required to explain the empirical $\sigma_{R}$ for nuclei such as ${ }^{11} \mathrm{Li}$. While this qualitative feature is not in doubt, there remain questions on the extent to which quantitative information can be determined. While some argue that measurements provide evidence of the neutron halo, requiring a density with a diffuse tail [5-10], others caution that cross sections are sensitive only to the matter rms radius $[11,12]$ and hence can be reproduced by a suitably chosen Gaussian density for the projectile.

It is therefore suggested that cross section measurements determine only an effective radius which may deviate from the rms radius if the density distribution deviates markedly from an assumed Gaussian or harmonic-oscillator singleparticle model distribution [9,13]. More microscopic studies, using multiple scattering Glauber approaches [11,14], also known as diffractive eikonal models, suggest that the optical limit Glauber model is rather poor for extended objects such as halo nuclei, even at energies of several hundred $\mathrm{MeV}$ per nucleon [15]. Other studies suggest that experimental reaction cross sections, even for exotic nuclei, can be reproduced using the Glauber model within the framework of nuclear transport theory [16].

In all cases, information about rms radii can only be meaningfully extracted from cross section measurements if some form is assumed for the radial density distributions of the projectile and target $[17,18]$. In principle one can learn more about the density distribution by measuring the reaction cross sections at several energies $[8,9,15,19]$ or on a range of targets [3]. Studies to determine the density distribution using both energy and target dependence of reaction cross section measurements have also been published $[6,7,20]$. In this paper we present a model study of the sensitivity of the nuclear part of the total reaction cross section to the assumed density distribution of ${ }^{11} \mathrm{Li}$ for a range of targets $\left(p,{ }^{12} \mathrm{C}\right.$, and $\left.{ }^{208} \mathrm{~Pb}\right)$ at $800 \mathrm{MeV} /$ nucleon.

\section{OPTICAL LIMIT GLAUBER MODEL}

Within the optical limit Glauber model, the total reaction cross section is written

$$
\sigma_{R}=2 \pi \int_{0}^{\infty} d b b[1-T(b)],
$$

where $T(b)$ is the transparency function at impact parameter $b$

$$
T(b)=\exp \left[-\sigma_{N N} \int d^{2} \mathbf{b}_{1} \rho_{P}^{(z)}\left(b_{1}\right) \rho_{T}^{(z)}\left(\left|\mathbf{b}-\mathbf{b}_{1}\right|\right)\right] .
$$

In Eq. (2) $\sigma_{N N}$ is the nucleon-nucleon cross section at the appropriate $N N$ relative energy and $\rho_{P}$ and $\rho_{T}$ refer to the projectile and target matter densities, respectively, with $\rho_{i}^{(z)}(b)$ the corresponding thickness functions, e.g., [19].

We will not address corrections to the optical limit model arising from the precise prescription used for the $N N$ cross section or other sources. Bertsch et al. [21]. discussed uncertainties in $\sigma_{N N}$ which translate into uncertainties in the deduced rms radius of order $0.1 \mathrm{fm}$ in the energy regime presented here. The folding of the target and projectile densities in the model above also implies a zero-range treatment of the $N N$ effective interaction and finite range effects can increase the calculated $\sigma_{R}$ by order of $10 \%$ [21]. These effects, while significant, will be present and of similar order in all the systems we consider. Our emphasis is not upon absolute values of calculated cross sections, but on the qualitative differences between calculations for systems with different target masses.

\section{DENSITY MODELS}

An analytic evaluation of the optical limit Glauber cross section can be performed [22] if one assumes Gaussian densities for the projectile and target nuclei. In this oneparameter density limit the only theoretical inputs are the rms radii of the interacting nuclei, to which the density range parameters are adjusted, and the $N N$ cross section. The Gaussian density is inappropriate for nuclei such as ${ }^{11} \mathrm{Li}$ whose matter distribution has an extended tail. Interaction cross section $\left(\sigma_{I}\right)$ data exist for ${ }^{11} \mathrm{Li}$ on proton and ${ }^{12} \mathrm{C}[6]$ and ${ }^{208} \mathrm{~Pb}$ [23] targets at $800 \mathrm{MeV} /$ nucleon. For ${ }^{11} \mathrm{Li}$ the 


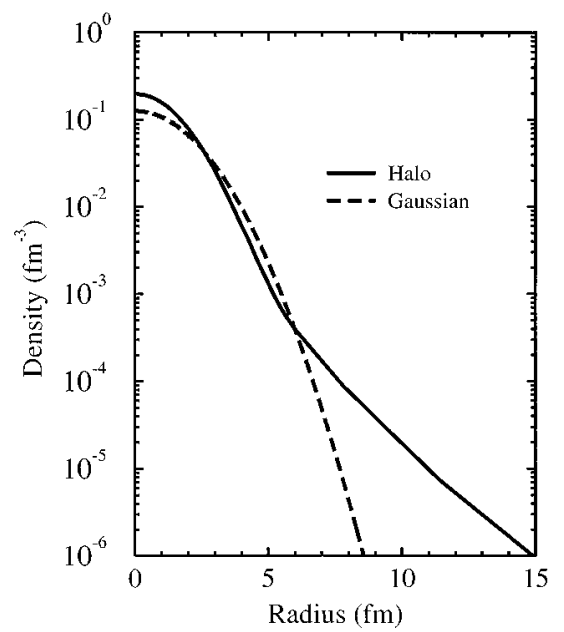

FIG. 1. Radial density distributions of the Faddeev three-body model and single Gaussian model of ${ }^{11} \mathrm{Li}$. Both have a rms radius $\left\langle r^{2}\right\rangle_{P}^{1 / 2}=3.04 \mathrm{fm}$.

interaction and reaction cross sections are essentially equal, thus we calculate $\sigma_{R}$ but compare with the experimental values of $\sigma_{I}$.

As a first orientation we consider two models for the density of the ${ }^{11} \mathrm{Li}$ projectile $\rho_{P}$ entering Eq. (2). The first, called here a halo density, is obtained from a three-body $\left({ }^{9} \mathrm{Li}\right.$ core $\left.+n+n\right)$ Faddeev equation solution of ${ }^{11} \mathrm{Li}$ (the L6A model prescription of [24]). The second density is a simple Gaussian with the same rms matter radius, $\left\langle r^{2}\right\rangle_{P}^{1 / 2}=3.04 \mathrm{fm}$. Figure 1 compares these radial density distributions. The calculated and experimental $\sigma_{R}$ for the ${ }^{11} \mathrm{Li}$ $+{ }^{12} \mathrm{C}$ and ${ }^{11} \mathrm{Li}+$ proton systems, at $800 \mathrm{MeV} /$ nucleon, are presented in Table I. Both densities essentially generate cross sections within quoted errors, particularly for the ${ }^{12} \mathrm{C}$ target. This example would suggest that the reaction cross section is determined, regardless of details of the density, by the matter rms radius. The suggestion, that cross section measurements provide an accurate means of determining rms radii, was made early in the analysis of such data [3] and neutron, proton, and matter rms radii have been quoted with considerable accuracy $[9,13]$ based on calculations which assume simple model densities.

We note in passing that for the proton target, contrary perhaps to intuition, the cross section from the more extended halo density is in fact smaller than that of the more compact Gaussian density. In the following we return to this effect in more detail and show that it is expected to be a quite general feature. Calculations from only two distinct densities, such as those above, or from a finite number of diverse models, does not allow one to easily elucidate this residual sensitivity. A simple model which allows a continuous variation in one or more features of the density is then of value.
The apparent dominant dependence of $\sigma_{R}$ upon the $r^{2}$ moment of the projectile density, $\left\langle r^{2}\right\rangle_{P}$, is certainly not readily deduced from Eqs. (1) and (2), except in the trivial case of a one-parameter description, such as a Gaussian model [22]. In this sense, a simple two-parameter description will already allow sufficient flexibility to maintain a given projectile $\left\langle r^{2}\right\rangle_{P}$ while adjusting another radial moment of the distribution, and so to examine residual sensitivities to this distribution. To this end, we study the dependence of the cross section on the $\left\langle r^{2}\right\rangle_{P}$ and $\left\langle r^{4}\right\rangle_{\mathrm{P}}$ moments by defining a ${ }^{11} \mathrm{Li}$ density as a sum of two terms of the form

$$
\rho_{\mathrm{P}}(r)=A_{c} e^{-r^{2} / a_{c}^{2}}+A_{v} r^{2} e^{-r^{2} / a_{v}^{2}}
$$

inspired by a harmonic-oscillator single-particle description. Here the first term represents the density of a mass 9 core and is normalized to nine. The second term simulates a longer range two-valence neutron component.

We use $\left\langle r^{2}\right\rangle_{\mathrm{P}}$ and $\left\langle r^{4}\right\rangle_{\mathrm{P}}$ as the two parameters of this distribution rather than $a_{c}$ and $a_{v}$. This is of course an arbitrary choice but these moments are instructive in the present context. We also require that $a_{c} \leqslant a_{v}$, in keeping with our physical picture of core and halo contributions.

\section{REACTION CALCULATIONS}

We present calculations for ${ }^{11} \mathrm{Li}$ induced reactions at 800 $\mathrm{MeV} /$ nucleon. Experimental reaction cross section data available at this energy include proton, ${ }^{12} \mathrm{C}[6]$, and ${ }^{208} \mathrm{~Pb}$ [23] targets. We will not attempt a detailed quantitative comparison with these data, in part for the reasons discussed earlier. Additionally, for heavier targets there are significant contributions to the cross section from electromagnetic dissociation (EMD) of the projectile; estimated [23] to be of order $30 \%$ for ${ }^{208} \mathrm{~Pb}$ but less than $2 \%$ for ${ }^{12} \mathrm{C}$. These contributions of multipole Coulomb forces are not included in the model calculations presented, which should be interpreted as the nuclear contribution to the cross section. Effects due the monopole Coulomb force are included using the modified impact parameter prescription of Charagi and Gupta [19] but are negligible at the energies of interest. We will show the empirical cross section values for the proton and ${ }^{12} \mathrm{C}$ targets on the appropriate figures only to clarify the magnitude of the effects calculated here in relation to the stated accuracy of available data.

Calculations are carried out by evaluating Eqs. (1) and (2) numerically. The $N N$ cross section used [19] is $41 \mathrm{mb}$. The ${ }^{12} \mathrm{C}$ density is assumed to be a single Gaussian distribution with an rms radius of $2.32 \mathrm{fm}$ [13]. The density for the ${ }^{208} \mathrm{~Pb}$ target was taken as a two-parameter Fermi form [25].

TABLE I. Experimental reaction cross sections at $800 \mathrm{MeV} /$ nucleon are compared with the values calculated using a Gaussian and halo density distribution.

\begin{tabular}{lccc}
\hline \hline Reaction & $\sigma_{R}(\exp )(\mathrm{mb})$ & $\sigma_{R}($ Gaussian $)(\mathrm{mb})$ & $\sigma_{R}($ halo $)(\mathrm{mb})$ \\
\hline${ }^{11} \mathrm{Li}+\mathrm{p}$ & $276 \pm 8$ & 278 & 259 \\
${ }^{11} \mathrm{Li}+{ }^{12} \mathrm{C}$ & $1056 \pm 14$ & 1065 & 1069 \\
\hline \hline
\end{tabular}




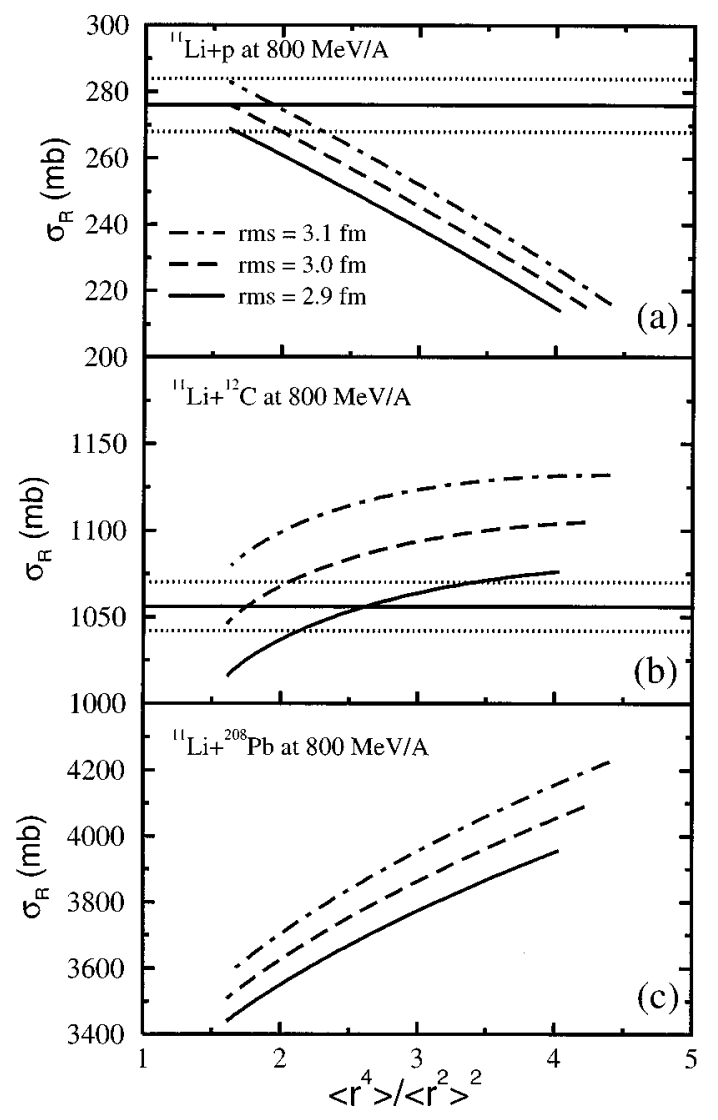

FIG. 2. Variation of $\sigma_{R}$ with $\left\langle r^{4}\right\rangle_{P} /\left\langle r^{2}\right\rangle_{P}^{2}$ at fixed values of $\left\langle r^{2}\right\rangle_{P}^{1 / 2}$ of $2.9 \mathrm{fm}$ (solid line), $3.0 \mathrm{fm}$ (dashed line), and $3.1 \mathrm{fm}$ (dot-dashed line). (a), (b), and (c) are for a proton, ${ }^{12} \mathrm{C}$, and ${ }^{208} \mathrm{~Pb}$ target.

\section{A. Reaction cross sections}

Figure 2 shows the variation of $\sigma_{R}$, for given $\left\langle r^{2}\right\rangle_{P}^{1 / 2}$ values, as a function of $\left\langle r^{4}\right\rangle_{P}$. Figures 2(a), (b), and (c) are for a proton, ${ }^{12} \mathrm{C}$, and ${ }^{208} \mathrm{~Pb}$ target, respectively. The curves actually show the variation of $\sigma_{R}$ with the ratio $\left\langle r^{4}\right\rangle_{P} /\left\langle r^{2}\right\rangle_{P}^{2}$ at the stated fixed values of $\left\langle r^{2}\right\rangle_{P}^{1 / 2}$ of $2.9 \mathrm{fm}$ (solid curve), 3.0 fm (dashed curve), and $3.1 \mathrm{fm}$ (dot-dashed curve). This particular ratio measure is a constant $(5 / 3)$ in the case of a single Gaussian density for ${ }^{11} \mathrm{Li}$ and would generate points on this fixed vertical line. For the two-parameter density each rms radius leads to a locus of points as shown. The curves cut off at $\left\langle r^{4}\right\rangle_{P} /\left\langle r^{2}\right\rangle_{P}^{2} \approx 1.6$ at which $a_{c}=a_{v}$. The loci show that within the assumed model there is no unique rms radius which generates a given reaction cross section, or, given a rms radius there are a range of density distributions consistent with empirical values and stated uncertainties.

Features of Fig. 2 of interest are the slopes of the $\sigma_{R}$ versus $\left\langle r^{4}\right\rangle_{P} /\left\langle r^{2}\right\rangle_{P}^{2}$ curves and their dependence upon the target mass/size. Specifically, the slopes of the curves for a proton and for ${ }^{12} \mathrm{C}$ and ${ }^{208} \mathrm{~Pb}$ targets, are of opposite sign. For the proton target increasing $\left\langle r^{4}\right\rangle_{P}$ decreases the reaction cross section and, on average, the ${ }^{11} \mathrm{Li}$ becomes more transparent. A hint of this feature was already noted in the calculations for the halo and Gaussian densities of Table I. For ${ }^{12} \mathrm{C}$ and ${ }^{208} \mathrm{~Pb}$ targets, Figs. $2(\mathrm{~b})$ and (c), the same projectile density changes have the opposite effect, $\sigma_{R}$ increasing with

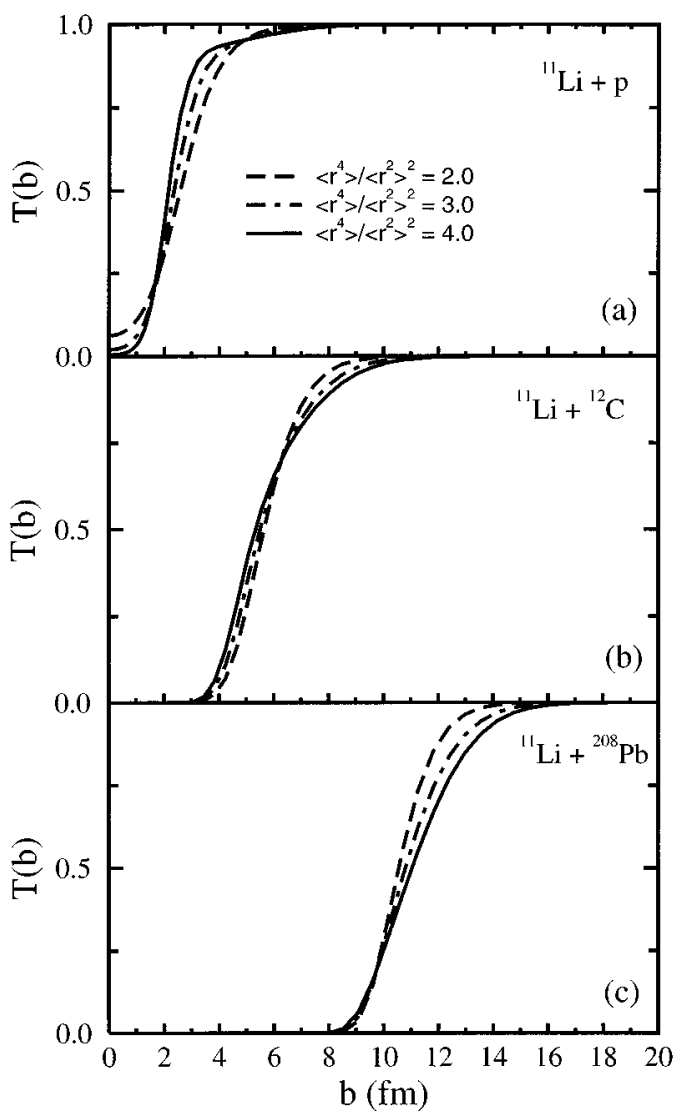

FIG. 3. Calculated transparency functions $T(b)$ as a function of impact parameter for $\left\langle r^{4}\right\rangle_{P} /\left\langle r^{2}\right\rangle_{P}^{2}=2.0$ (dashed), 3.0 (dot-dashed), and 4.0 (solid curve), at fixed projectile rms radius $\left\langle r^{2}\right\rangle_{P}^{1 / 2}(3.0 \mathrm{fm})$. (a), (b), and (c) are for a proton, ${ }^{12} \mathrm{C}$, and ${ }^{208} \mathrm{~Pb}$ target.

$\left\langle r^{4}\right\rangle_{P}$ at fixed $\left\langle r^{2}\right\rangle_{P}$. Additionally, the sensitivity to $\left\langle r^{4}\right\rangle_{P}$ is different for the two heavier targets, the ${ }^{208} \mathrm{~Pb}$ target showing much greater fractional changes in $\sigma_{R}$.

We comment that an increased $\left\langle r^{4}\right\rangle_{P}$ requires a more localized core density at constant $\left\langle r^{2}\right\rangle_{P}$, so there is an interplay between the effects of increasing $a_{v}$ and decreasing $a_{c}$. A more extended valence distribution due to an increased $a_{v}$ results, at all impact parameters, in the target overlapping the ${ }^{11} \mathrm{Li}$ halo over a greater distance along its assumed straight line path. At large impact parameters therefore the transparency of the collision will be reduced. On the other hand the reduced density of the halo results in a smaller probability that the target will encounter a valence nucleon for a range of smaller impact parameter values. The final outcome of this interplay is obtained by correctly folding the thickness functions $\rho_{i}^{(z)}(b)$, as is described by Eq. (2). These features are clarified by reference to the transparency functions $T(b)$, entering the integrand in Eq. (1), and the integrands $b[1-T(b)]$ themselves.

\section{B. Transparency functions}

To clarify the different gradients of the $\sigma_{R}$ curves in Fig. 2, in Fig. 3 we show the $T(b)$, at fixed $\left\langle r^{2}\right\rangle_{P}^{1 / 2}$ (3.0 fm), for $\left\langle r^{4}\right\rangle_{P} /\left\langle r^{2}\right\rangle_{P}^{2}=2.0$ (dashed curve), 3.0 (dot-dashed curve), and 4.0 (solid curve). Figure 4 shows their contribution to the reaction cross section, the integrands $b[1-T(b)]$ ap- 


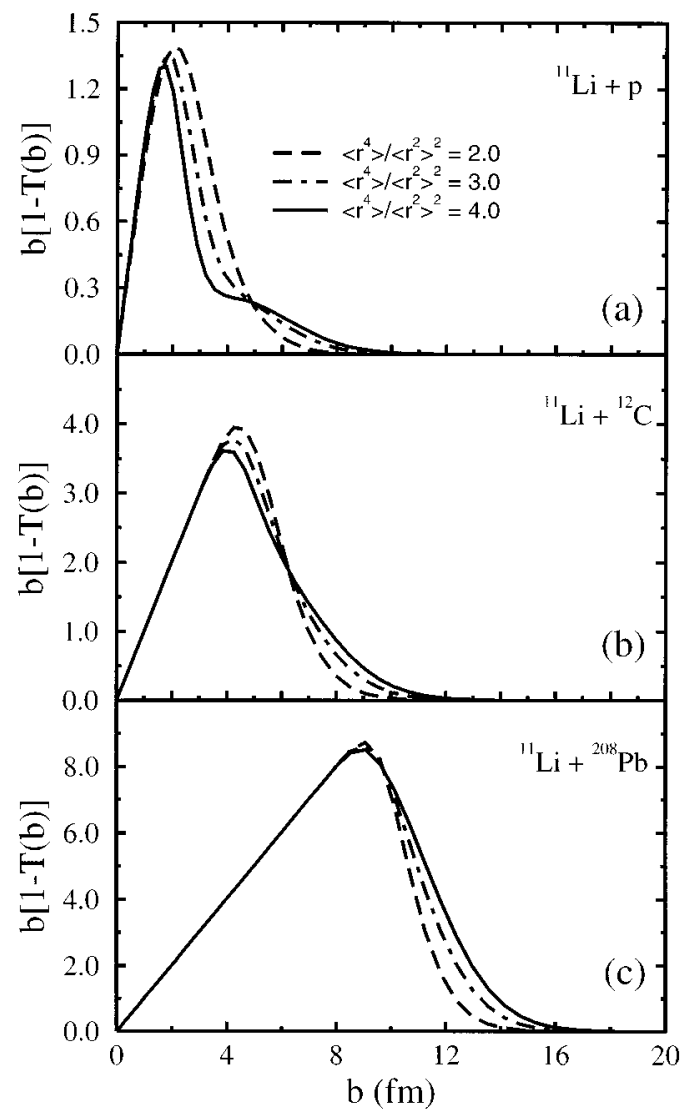

FIG. 4. Calculated $\sigma_{R}$ integrands $b[1-T(b)]$ as a function of impact parameter for $\left\langle r^{4}\right\rangle_{P} /\left\langle r^{2}\right\rangle_{P}^{2}=2.0$ (dashed), 3.0 (dot-dashed), and 4.0 (solid curve), at fixed projectile rms radius $\left\langle r^{2}\right\rangle_{P}^{1 / 2}(3.0 \mathrm{fm})$. (a), (b), and (c) are for a proton, ${ }^{12} \mathrm{C}$, and ${ }^{208} \mathrm{~Pb}$ target.

pearing in Eq. (1), for the same densities. (a), (b), and (c) of each figure is for a proton, ${ }^{12} \mathrm{C}$, and ${ }^{208} \mathrm{~Pb}$ target, respectively.

To a reasonable first approximation, except in the limit of large $b$ on the proton target we can approximate $[1-T(b)]$ by the Fermi distribution

$$
1-T(b)=\left\{1+\exp \left[\left(b-b_{0}\right) / a\right]\right\}^{-1},
$$

where $b_{0}$ is the strong absorption impact parameter, $T\left(b_{0}\right)=1 / 2$, and $a$ a surface diffuseness measure. This connects analytically $\sigma_{R}$ with the transparency function and to order $\left(a / b_{0}\right)^{2},[26]$

$$
\sigma_{R}=\pi b_{0}^{2}\left[1+\pi a^{2} /\left(3 b_{0}^{2}\right)\right]
$$

where from Fig. 3 the $b_{0}$ and $a$ vary with $\left\langle r^{4}\right\rangle_{P}$.

For a proton target, Fig. 3(a), the dominant trend is that both $b_{0}$ and $a$ decrease with increasing $\left\langle r^{4}\right\rangle_{P}$, leading to a falling cross section. More precisely, Fig. 4(a) shows that the changes in the $T(b)$ result in a small and reduced surface transparency at the largest contributing $b$ values but the major effect is a greater transparency at impact parameters between 3 and $5 \mathrm{fm}$. This results from the combination of a reduced range of the core distribution $\left(a_{c}\right)$ and of the valence density being forced to larger radii with increasing $a_{v}$. The resulting $\sigma_{R}$, Fig. 2(a), falls by approximately $20 \%$ as $\left\langle r^{4}\right\rangle_{P} /\left\langle r^{2}\right\rangle_{P}^{2}$ varies from 2.0 to 4.0 at constant $\left\langle r^{2}\right\rangle_{P}^{2}$. The available experimental interaction cross section $\sigma_{I}$ datum [6] is shown by the horizontal band, with an error of order $3 \%$.

For the ${ }^{208} \mathrm{~Pb}$ target, Fig. 3(c), the situation is also rather clear. Both $b_{0}$ and $a$ increase with $\left\langle r^{4}\right\rangle_{P}$, causing $\sigma_{R}$ to increase. Figure 4(c) shows this results from a reduced surface transparency to the heavy target. The large target is unable to discern finer details of the core and valence distributions at the smaller impact parameters and the reduced range of the core distribution plays no obvious role in this case. The calculated $\sigma_{R}$, Fig. 2(c), increase by $20 \%$ as $\left\langle r^{4}\right\rangle_{P} /\left\langle r^{2}\right\rangle_{P}^{2}$ varies from 2.0 to 4.0 with $\left\langle r^{2}\right\rangle_{P}^{2}$ held fixed. The experimental value in this case, $5380 \pm 640 \mathrm{mb}$ [23], includes a large electromagnetic dissociation (EMD) component, and is not shown. Nuclear contributions to the cross section, as discussed here, have previously been used to estimate the magnitude of these EMD contributions by subtraction from the empirical cross section [23,27]. Such analyses assume projectile $\left\langle r^{2}\right\rangle_{P}$ values deduced from data on lighter targets, where EMD effects can reasonably be neglected. Since the present work shows the nuclear cross sections for heavy systems retain quite considerable residual sensitivity to the projectile density, beyond its $\left\langle r^{2}\right\rangle_{P}$ value, conclusions regarding the magnitude of these EMD contributions on high $Z$ targets will also be significantly model dependent.

For the ${ }^{12} \mathrm{C}$ target, Fig. 3(b), the changes in the shapes of the $T(b)$ with $\left\langle r^{4}\right\rangle_{P}$ are intermediate between the proton and ${ }^{208} \mathrm{~Pb}$ situations, and more complicated. Although $b_{0}$ decreases with increasing $\left\langle r^{4}\right\rangle_{P}, a$ increases. The full calculations, and Fig. 4(b), show the effect of increasing $a$ wins over the effect of decreasing $b_{0}$, and $\sigma_{R}$ increases rather weakly with $\left\langle r^{4}\right\rangle_{P}$. The sensitivity in the calculated cross sections, Fig. 2(b), is less than that for the proton and the ${ }^{208} \mathrm{~Pb}$ targets and rise by approximately $5 \%$ as $\left\langle r^{4}\right\rangle_{P} /\left\langle r^{2}\right\rangle_{P}^{2}$ varies between 2.0 and 4.0. The available experimental interaction cross section $\sigma_{I}$ datum [6] is again shown by the horizontal band, with an error of order $1.3 \%$.

By performing additional calculations assuming a single Gaussian density for the target, such as used in the ${ }^{12} \mathrm{C}$ case above, one moves continuously between the ${ }^{12} \mathrm{C}$ and proton situations described above as the size of the target is adjusted. Thus the slope effects observed are a very general consequence of the geometrical target-projectile density overlaps. The observed sensitivity of calculated cross sections to the extension of the projectile one-body density is potentially valuable in setting empirical limits upon this distribution. The reasonably steep gradient in Fig. 2(a), means that simultaneously reproducing data on a proton and on another light target will place more severe constraints on the allowed density distribution; the values of $\left\langle r^{2}\right\rangle_{P}$ and $\left\langle r^{4}\right\rangle_{P}$ within our model. The reduced sensitivity to $\left\langle r^{4}\right\rangle_{P}$ in the case of the ${ }^{12} \mathrm{C}$ target might indicate this target is best suited to determine $\left\langle r^{2}\right\rangle_{P}^{1 / 2}$ empirically, however this ${ }^{12} \mathrm{C}$ result, being an intermediate situation between the light and heavy target limits, is more sensitive to the details of the model densities assumed. The ability to use data for heavy systems, such as the ${ }^{208} \mathrm{~Pb}$ target, in this way would add additional constraints, given its oppositely directed sensitivity. Use of these data would however first require a reliable means of estimating the EMD component of the cross section. 


\section{DISCUSSION AND CONCLUSIONS}

Model calculations of ${ }^{11} \mathrm{Li}$ induced reactions using the optical limit Glauber model show that the nuclear contributions to the reaction cross sections are sensitive to moments of the projectile density distribution other than the rms radius. We have explored these sensitivities using a simple, flexible, physically inspired, two-parameter projectile density which allows a variation of radial density distributions at a fixed rms radius $\left\langle r^{2}\right\rangle_{P}^{1 / 2}$. Our choice of a second density measure was the projectile $r^{4}$ radial moment, $\left\langle r^{4}\right\rangle_{P}$, for the study of the extended ${ }^{11} \mathrm{Li}$ system.

We have shown that the calculated cross section sensitivity to the valence nucleon extension is strongly dependent on the size of the target nucleus. For a ${ }^{12} \mathrm{C}$ target, extending the tail of the density can produce a change in the calculated $\sigma_{R}$ of order $5 \%$, to be compared with a quoted experimental error of order $2 \%$ or less on current experimental data. For a proton or a ${ }^{208} \mathrm{~Pb}$ target, the cross section sensitivity is considerably greater. Within the simple parametrization we have assumed, changes of up to $20 \%$ in $\sigma_{R}$ were obtained for projectile densities with the same $\left\langle r^{2}\right\rangle_{P}^{1 / 2}$. An important feature of the results is that these higher sensitivities to the $\left\langle r^{4}\right\rangle_{P}$ moment, the slopes of the cross section plots with $\left\langle r^{4}\right\rangle_{P}$, are in the opposite sense in the proton and heavy target limits. The latter sensitivity has implications for the magnitude of deduced electromagnetic dissociation contributions to cross sections on high $Z$ targets.

The results indicate that there is the potential, if calculations and data for proton and light target systems are taken together, to make a more precise assessment of the projectile rms radius. Alternatively, if the rms radius is known independently, then loci such as those in Fig. 2, which are readily calculated for more sophisticated model densities than has been assumed here, could be used to begin to place limits upon higher radial moments of the projectile density distributions. It should be noted that the phenomenon discussed here appears as a natural consequence of the folding of the projectile and target densities implied by the optical limit theory. It would be very interesting to understand the extent to which these model predictions are also manifest in calculations of cross sections using explicitly few-body Glauber model approaches which go beyond the optical limit theory.

\section{ACKNOWLEDGMENTS}

The financial support of the United Kingdom Engineering and Physical Sciences Research Council (EPSRC) in the form of Grants GR/J95867 and GR/K33026 and a research studentship (for M.P.B.) is gratefully acknowledged. We acknowledge useful discussions with Professor M. V. Zhukov in the early stages of this work.
[1] R.J. Glauber, in Lectures in Theoretical Physics, edited by W.E. Brittin (Interscience, New York, 1959), Vol. 1, p. 315.

[2] W. Czyz and L.C. Maximon, Ann. Phys. (N.Y.) 52, 59 (1969).

[3] I. Tanihata, H. Hamagaki, O. Hashimoto, Y. Shida, N. Yoshikawa, K. Sugimoto, O. Yamakawa, T. Kobayashi, and N. Takahashi, Phys. Rev. Lett. 55, 2676 (1985).

[4] I. Tanihata et al., Phys. Lett. 160B, 380 (1985).

[5] I. Tanihata, Nucl. Phys. A488, 113c (1988).

[6] I. Tanihata et al., Proceedings of the Second International Conference on Radioactive Nuclear Beams, Louvain-laNeuve, Belgium, 1991 (unpublished), p. 221; Phys. Lett. B 287, 307 (1992).

[7] S. Shimoura, Proceedings of the Second International Conference on Radioactive Nuclear Beams, Louvain-la-Neuve, Belgium, 1991 (unpublished), p. 215.

[8] M. Fukuda et al., Phys. Lett. B 268, 339 (1991).

[9] A. Ozawa et al., Phys. Lett. B 334, 18 (1994).

[10] H. Sato and Y. Okuhara, Phys. Lett. 162B, 217 (1985).

[11] Y. Suzuki, T. Kido, Y. Ogawa, K. Yabana, and D. Baye, Nucl. Phys. A567, 957 (1994).

[12] K. Sugimoto and I. Tanihata, Institute of Physical and Chemical Research (RIKEN) Report No. RIKEN-AF-NP-131 (unpublished).

[13] I. Tanihata, T. Kobayashi, O. Yamakawa, S. Shimoura, K.
Ekuni, K. Sugimoto, N. Takahashi, T. Shimoda, and H. Sato, Phys. Lett. B 206, 592 (1988).

[14] Y. Ogawa, K. Yabana, and Y. Suzuki, Nucl. Phys. A543, 722 (1992).

[15] N. Takigawa, M. Ueda, M. Kuratani, and H. Sagawa, Phys. Lett. B 288, 244 (1992).

[16] Y.G. Ma, W.Q. Shen, J. Feng, and Y.Q. Ma, Phys. Rev. C 48, 850 (1993).

[17] H.O. Meyer, Phys. Rev. C 17, 1116 (1978).

[18] K. Riisager, Ph.D. Thesis, University of Aarhus, 1994, unpublished.

[19] S.K. Charagi and S.K. Gupta, Phys. Rev. C 41, 1610 (1990).

[20] A.K. Chaudhuri, Phys. Rev. C 49, 1603 (1994).

[21] G.F. Bertsch, B.A. Brown, and H. Sagawa, Phys. Rev. C 39, 1154 (1989).

[22] P.J. Karol, Phys. Rev. C 11, 1203 (1974).

[23] T. Kobayasi et al., Phys. Lett. B 232, 51 (1989).

[24] M.V. Zhukov, D.V. Fedorov, B.V. Danilin, J.S. Vaagen, J.M. Bang, and I.J. Thompson, Nucl. Phys. A552, 353 (1993).

[25] R.C. Barrett and D.F. Jackson, Nuclear Sizes and Structure (Clarendon Press, Oxford, 1979), Table 2.3, p. 47.

[26] L.R.B. Elton, Nuclear Sizes (Oxford University Press, New York, 1961), p. 106.

[27] G.F. Bertsch, H. Esbensen, and A. Sustich, Phys. Rev. C 42, 758 (1990) 